\title{
How a small quantum bath can thermalize long localized chains
}

\author{
David J. Luitz, ${ }^{1}$ François Huveneers, ${ }^{2}$ and Wojciech De Roeck ${ }^{3}$ \\ ${ }^{1}$ Department of Physics T42, Technische Universität München, \\ James-Franck-Straße 1, 85748 Garching, Germany* \\ ${ }^{2}$ Université Paris-Dauphine, PSL Research University, CNRS, CEREMADE, 75016 Paris, France ${ }^{\dagger}$ \\ ${ }^{3}$ Instituut voor Theoretische Fysica, KU Leuven, Belgium ${ }^{\ddagger}$
}

(Dated: May 30, 2017)

\begin{abstract}
We investigate the stability of the many-body localized (MBL) phase for a system in contact with a single ergodic grain, modelling a Griffiths region with low disorder. Our numerical analysis provides evidence that even a small ergodic grain consisting of only 3 qubits can delocalize a localized chain, as soon as the localization length exceeds a critical value separating localized and extended regimes of the whole system. We present a simple theory, consistent with the arguments in [Phys. Rev. B 95, 155129 (2017)], that assumes a system to be locally ergodic unless the local relaxation time, determined by Fermi's Golden Rule, is larger than the inverse level spacing. This theory predicts a critical value for the localization length that is perfectly consistent with our numerical calculations. We analyze in detail the behavior of local operators inside and outside the ergodic grain, and find excellent agreement of numerics and theory.
\end{abstract}

Introduction - The phenomenon of Many-Body Localization (MBL) [1-15] has challenged our ideas on thermalization and the applicability of thermodynamics. It is hence important to determine the precise conditions for the stability of the MBL phase. Whereas in the original works $[3,4]$, the spatial dimension $d$ did not play a central role, the rigorous treatment of Griffiths regions of low disorder[16] in [17] relies on $d=1$, not for technical but for conceptual reasons. More generally, it is now well understood that there is a huge variety of systems where thermalization is effectively inhibited locally and only rare Griffiths regions can, possibly, restore ergodicity $[18,19]$. This applies in particular to quasilocalization [20-26], classical disordered models [27, 28] or even glasses $[29,30]$. It is also believed that Griffiths regions drive the transition from MBL to ergodicity [31-36]. This raises the fundamental issue of understanding the effect of a Griffiths region, in practice an ergodic grain or imperfect bath, on a localized system. We outline a very simple theory: local ergodicity characterized by the Eigenstate Thermalization Hypothesis (ETH) [37-43] is taken as the default option, and a degree of freedom is interpreted as localized if the local relaxation time would be larger than the inverse level spacing. In [44] a more microscopically motivated version of this theory was proposed leading to precisely the same conclusions. The main result is the instability of MBL if the bare localization length is larger than a critical value [45]. This predicted instability implies that a single, sufficiently large, interacting, ergodic grain thermalizes the whole system if the localization length in the localized part of the system is large enough. This striking conclusion is counter-intuitive, and it has often been suggested to us, e.g. [46], that localization should prevail when the number of a priori localized degrees of freedom clearly exceeds the number of degrees of freedom in the bath. In this letter, we investigate this aspect, using

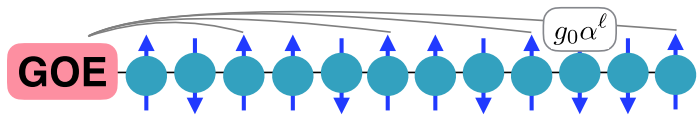

Figure 1. LIOMs (blue) coupled to an ergodic grain (red) modelled by a GOE matrix.

a setup where the ergodic grain is considerably smaller than the surrounding localized system, namely 3 versus 13 spins. All results of our numerical analysis confirm the simple theory, leading to thermalization of the chain by a small ergodic grain.

Theoretical predictions - Let us consider a system of size $L=L_{\mathrm{loc}}+L_{b}$ containing an ergodic grain (or bath) of size $L_{b}$ interacting with a fully localized chain of size $L_{\mathrm{loc}}$. The full system is described by the Hamiltonian $H=H_{b}+H_{\mathrm{loc}}+H_{b l}$. We assume that the bath Hamiltonian $H_{b}$ satisfies the eigenstate thermalization hypothesis (ETH). $H_{\text {loc }}$ describes an Anderson insulator in the basis of localized integrals of motion (LIOMs) [5, 15, 47-54], and $H_{b l}$ couples the bath locally to the LIOMs. A LIOM at distance $\ell$ from the bath is connected to the bath by a coupling strength $g_{\ell}:=g_{0} e^{-\ell / \xi}=g_{0} \alpha^{\ell}$ reminiscent of the interaction of an MBL system with the grain, where $\alpha=e^{-1 / \xi}$ and where $\xi$ denotes the localization length (in lattice units); cf. Fig. 1 and Eq. (5) for the model used in the numerics.

ETH provides an ansatz for the matrix elements of a local operator $A$ in an ergodic system. Let $E, E^{\prime}$ label eigenstates with energy density $\epsilon_{0}=E / L \sim E^{\prime} / L$, and let $\langle A\rangle_{\epsilon_{0}}=0$ (ensemble average in an equilibrium state at $\left.\epsilon_{0}\right)$. Below we always let $\epsilon_{0}$ correspond to maximal entropy and we drop $\epsilon_{0}$ from the notation. Then, ETH amounts to [42]

$$
\left\langle E^{\prime}|A| E\right\rangle=\sqrt{\delta} f(\omega) \eta_{E, E^{\prime}} \quad\left(E \neq E^{\prime}\right)
$$

with $\delta$ the many-body level spacing at density $\epsilon_{0}, \omega=$ 
$E-E^{\prime}$ the energy difference, $\eta_{E, E^{\prime}}$ random numbers with zero mean and unit variance and $f(\omega)$ a smooth function that can be related to a time-dependent correlation function and that satisfies the sum rule $\int d \omega|f(\omega)|^{2}=$ $\left\langle A^{\dagger} A\right\rangle_{\epsilon_{0}} \sim 1$. For local $A, f(\omega)$ is roughly supported on intervals of size $\Delta$, where $\Delta$ can be interpreted as the typical rate at which local quantities equilibrate $[42,55,56]$ [57]. Obviously, the ansatz (1) only makes sense if $\Delta \gg \delta$ (otherwise (1) conveys no information), which we use as a consistency condition that will be invoked below in a crucial way. Let us give a relevant example of how to determine $\Delta$ : take one LIOM with field $h$ coupled to an ergodic system (e.g. our ergodic grain) via a weak coupling term of strength $g$. Then, under some mild conditions, Fermi's Golden Rule predicts $f(\omega)$ of that LIOM in the combined system to have principal peaks at the Bohr frequencies $\pm 2 h$ with widths of order $\Delta=g^{2} / \epsilon$, for some local energy scale $\epsilon$ characterizing the bath, see e.g. [44].

Now, we couple LIOMs $i=1, \ldots, L_{\mathrm{loc}}$ to the bath ( $c f$. Fig. 1) with couplings $g_{i}=g_{0} \alpha^{i}$ and compute the local rates $\Delta_{i}$ as in the example above, namely $\Delta_{i} \sim \alpha^{2 i} g_{0}^{2} / \epsilon$. Now we assume that ETH is valid as long as it is not inconsistent, i.e. as long as $\delta \ll \Delta_{i}$ for all $i \leq L_{\text {loc. This }}$ central assumption will lead to striking consequences, presented below. Verifying these consequences is the main point of the paper. We conjecture hence that ETH for the full system is valid if and only if

$$
\left(g_{0}^{2} / \epsilon\right) \alpha^{2 L_{\mathrm{loc}}} \geq \mathcal{W} 2^{-L},
$$

where we put $\delta=\mathcal{W} 2^{-L}$ with $\mathcal{W}=\epsilon_{0} L$ the spectral width. Neglecting all non-exponential dependence on the lengths $L_{\mathrm{loc}}, L_{b}$, the condition for full ETH becomes (leading order in $L_{b} \gg 1$ )

$$
L_{\mathrm{loc}} \leq L_{c}=\frac{L_{b} \log 2}{\log \alpha^{-2}-\log 2}
$$

The most striking consequence of this analysis is that $L_{c}=\infty$ if $\alpha>\alpha_{c}=\frac{1}{\sqrt{2}}$ (and $L_{b}$ is not too small). That is: a small bath is capable of thermalizing an arbitrary number of LIOMs. This is a sharp prediction that can be tested numerically by studying the validity of ETH as a function of $\alpha$.

Let us add a more quantitative prediction of the theory: The local rates $\Delta_{i}$ correspond to an effective dimension $d_{i}=\Delta_{i} / \delta$, i.e. the number of eigenstates $\left|E^{\prime}\right\rangle$ over which $A_{i}|E\rangle$ is spread out, according to (1), for a local operator $A_{i}$ at site $i$. Then our theory predicts that

$$
d_{i} \approx 2^{L_{\text {therm }}} \alpha^{2 i}, \quad L_{\text {therm }}=L_{b}+\min \left(L_{\mathrm{loc}}, L_{c}\right),
$$

as long as $d_{i} \geq 1$, and setting $d_{i}=1$ otherwise, corresponding to localization at site $i$. Here, $L_{\text {therm }}$ is the length of the thermal region and for $\alpha>\alpha_{c}$, we simply have $L_{\text {therm }}=L$. In the rest of this letter, we present the
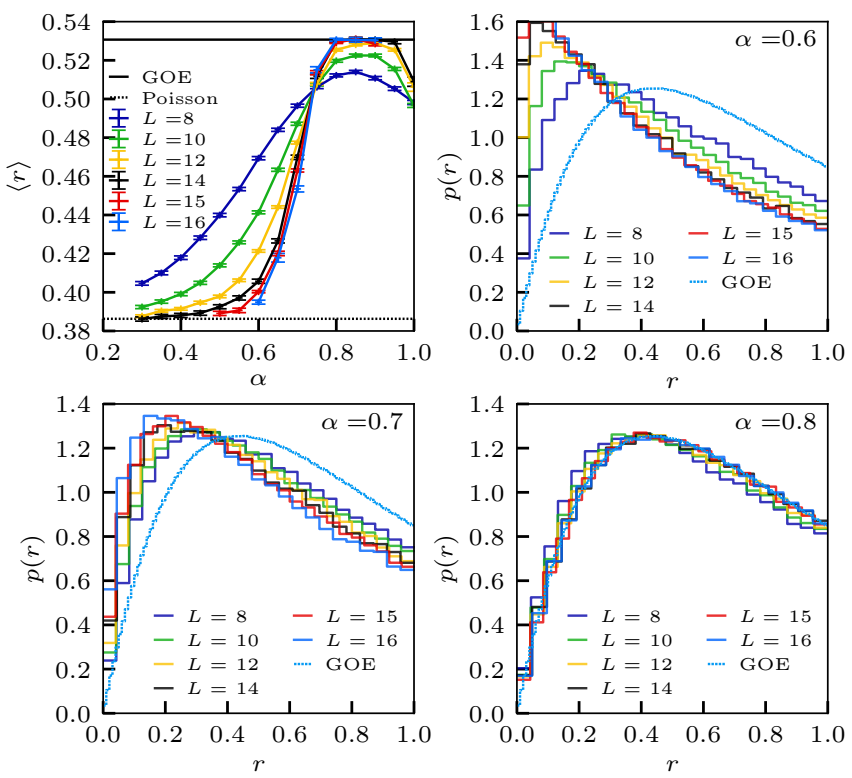

Figure 2. Probability distributions and average of the adjacent gap ratio $r$ for different coupling strengths and system sizes compared to the distribution obtained for large GOE matrices.

several numerical results that demonstrate the accuracy of the above picture for numerically accessible system sizes.

Model - We start from a simplified model of an ergodic grain coupled to localized spins, as depicted on Fig. 1, where the Hamiltonian of the ergodic grain is given by a random matrix $\mathrm{R}$ drawn from the GOE ensemble: $\mathrm{R}=\frac{\beta}{2}\left(\mathrm{~A}+\mathrm{A}^{T}\right) \in \mathbb{R}^{2^{L_{b}} \times 2^{L_{b}}}$ where $\mathrm{A}_{i j}=\operatorname{norm}(0,1)$ with norm $(0,1)$ random numbers drawn from a normal distribution with zero mean and unit variance.

Now, we can specify the Hamiltonian for our model for an ergodic grain of size $L_{b}$ and a set of LIOM's on sites $0, \ldots, L_{\mathrm{loc}}-1$.

$$
H=\mathrm{R}+\sum_{i=0}^{L_{\mathrm{loc}}-1} \frac{h_{i}}{2} \sigma_{i}^{z}+\sum_{i=0}^{L_{\mathrm{loc}}-1} \frac{g_{0} \alpha^{i}}{4} \sigma_{i}^{x} \sigma_{-1}^{x} .
$$

The system has no conservation laws except energy and the total Hilbert space dimension is $\operatorname{dim}(\mathcal{H})=2^{L}$. We restrict the size of the ergodic grain to $L_{b}=3$ (on sites $-3,-2,-1$, hence $\sigma_{-1}^{x}$ is a bath operator). For a more accurate correspondence of the LIOMs to a generic MBL system, we would need to include interactions of the type $\sigma_{i}^{z} \sigma_{i+1}^{z}$. For simplicity, we omit those interactions, thus making our localized chain basically an Anderson Insulator (AI). This simplification does not change the physics, since anyhow the bath coupling makes the full system truly interacting. Our theory is hence not in conflict with the fact that one can construct AIs with arbitrarily large localization lengths.

The onsite fields $h_{i}$ are drawn from a random box distribution $[1-W, 1+W], W=0.5$. The shift of the 
box distribution by 1 is not necessary but reduces finite size effects. We have checked carefully that a symmetric distribution around 0 yields similar results. In all our experiments, $L_{b}=3, \beta=0.3, g_{0}=1$. We have selected these values so as to obtain the cleanest results with the smallest bath size and the smallest coupling constant $g_{0}$, however our results remain qualitatively similar if any of these three parameters is varied (with $L_{b} \geq 3$ ). In order to infer the behavior of the system in the thermodynamic limit, we vary the number $L_{\mathrm{loc}}$ of localized spins coupled to the bath, with $L_{\mathrm{loc}} \leq 13$. For $L_{\mathrm{loc}}=13$, the coupling strength of the last spin is $g_{\min }=g_{0} \alpha^{12}$. For $\alpha=0.8>\alpha_{c}$, the direct coupling to the bath is $0.8^{12} \approx 0.068$ and does not suffice to trivially thermalize the last few spins ( $c f$. Supplemental Material). Thus, for such values of $\alpha$, the thermalization of the last spins results from highly non-trivial effects involving all spins in between.

Spectral statistics - A powerful and very general measure of ergodicity of quantum systems are the statistical properties of its energy spectrum, typically studied in the center of the spectrum. The gap ratio[6, 7] $r=\min \left\{\Delta E_{k}, \Delta E_{k+1}\right\} / \max \left\{\Delta E_{k}, \Delta E_{k+1}\right\}$ with $\Delta E_{k}=E_{k+1}-E_{k}$ is Poisson distributed for localized systems and GOE distributed for ergodic ones, as an account of level repulsion [58]. In Fig. 2, we show the average of $r$ as a function of $\alpha$ for various system sizes $L$. A clear tendency towards GOE statistics for $\alpha \gtrsim 0.7$ and towards Poisson statistics for $\alpha \lesssim 0.7$ is visible, consistently with the theoretical value for $\alpha_{c} \approx 0.7071$. It is important to note that in the supercritical regime, the increase of $\langle r\rangle$ with $L$ is due to the addition of increasingly weakly coupled spins. To get a more detailed picture near the transition, we show the full distribution of $r$ for $\alpha=0.6,0.7,0.8$ : For $\alpha=0.6,0.7$ we observe a clear drift towards Poisson statistics as the system sizes increases, while no deviation from the GOE statistics is observed at $\alpha=0.8$ up to $L=16$ [59].

Local magnetizations - A direct test of the validity of ETH is furnished by the diagonal values of a local operator (here $\sigma_{i}^{z}$ ) in the eigenbasis of the Hamiltonian: $\left\langle E\left|\sigma_{i}^{z}\right| E\right\rangle$. In a localized system, the distribution of $\left\langle E\left|\sigma_{i}^{z}\right| E\right\rangle$ is sharply bimodal with peaks at \pm 1 , since the LIOMs are small perturbations of the bare spins $\sigma_{i}^{z}$. For an ergodic system, the distribution is sharply peaked around the thermal value (here: 0 ), with variance scaling $[60,61]$ as $1 / d_{i}$ with $d_{i}$ the effective dimension, as computed in Eq. (4). To test this, we show in Fig. 3 (top) the standard deviation over disorder and all eigenstates $|E\rangle$ in a small window at maximal entropy of the expectation values $\left\langle E\left|\sigma_{i}^{z}\right| E\right\rangle$, as a function of site index $-3 \leq i<L_{\mathrm{loc}}$ for $\alpha=0.6<\alpha_{c}$ and $\alpha=0.8>\alpha_{c}$. At $\alpha=0.6$, we observe that the standard deviation goes down slightly for $i<0$ as we start increasing $L$ but saturates quickly to a constant value. This indicates that the first spins near the bath are thermalized and increase
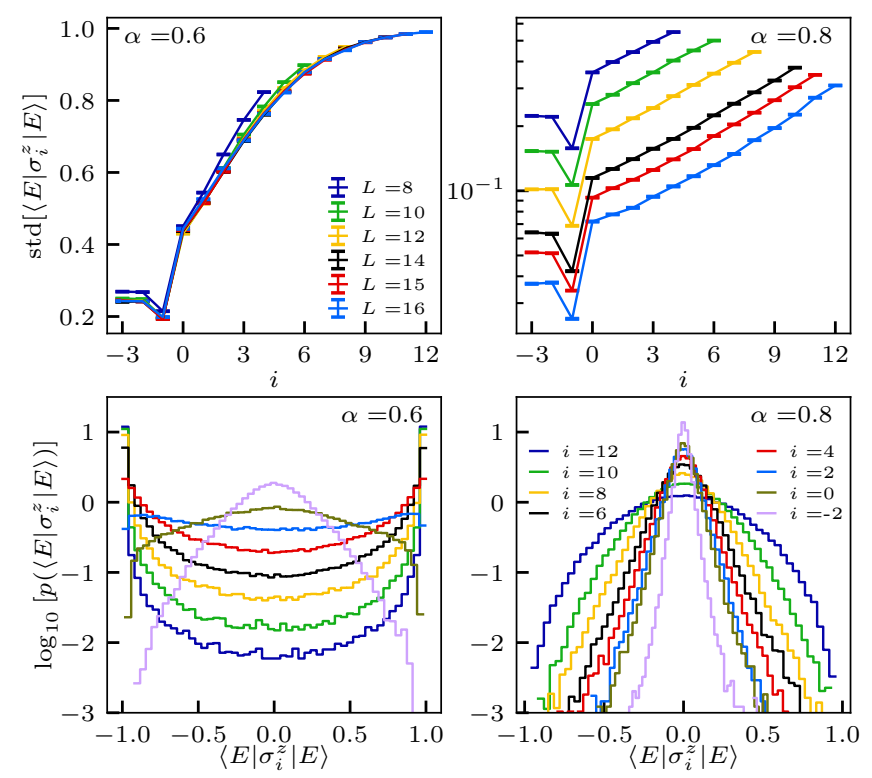

Figure 3. Top: Average over disorder realizations of the standard deviation of the eigenvector expectation of the magnetization at site $i(i<0$ is in the bath, and $i \geq 0$ are LIOMs) for various total length $L$. Bottom: Distribution of eigenvector expectation values of local magnetizations for $L=16$ for different positions in the chain.

the effective dimension for local operators in the bath, while spins further away, at $i>L_{c}$, remain localized and do not affect the effective dimension. For $i \geq 0$, the standard deviation tends to its maximal value 1 as the operator moves away from the bath, consistently with the fact that only the closest spins get thermalized. The very good data collapse at large $L$ indicates that stationary values have been reached. The situation is strikingly different at $\alpha=0.8$ : at any fixed distance $i$ from the bath, the standard deviation decreases as the total length $L$ increases, because all spins contribute to the effective dimension. On the other hand, for fixed $L$, the standard deviation always increases as one moves away from the bath (i.e. as $i$ increases). This is fully consistent with the decreasing effective dimension predicted in (4) and it should hence not be interpreted as some sort of "imperfect thermalization". Finally, it is instructive to compare the standard deviation of the last spin at different $L$, i.e. the endpoints of the curves: these endpoints move down with increasing $L$. Thus, the last spins become more and more delocalized, even though they also move further away from the original bath.

This picture is even clearer in the full distribution of $\left\langle E\left|\sigma_{i}^{z}\right| E\right\rangle$ shwon for $L=16$ for a subset of sites $i$ and $\alpha=0.6,0.8$ in Fig. 3 (bottom). At $\alpha=0.6$, the distribution of all but the first few spins become strongly bimodal with peaks as \pm 1 , indicating that spins far away form the bath are indeed not thermalized. In contrast, for $\alpha=0.8$, we see a progressive broadening of the dis- 


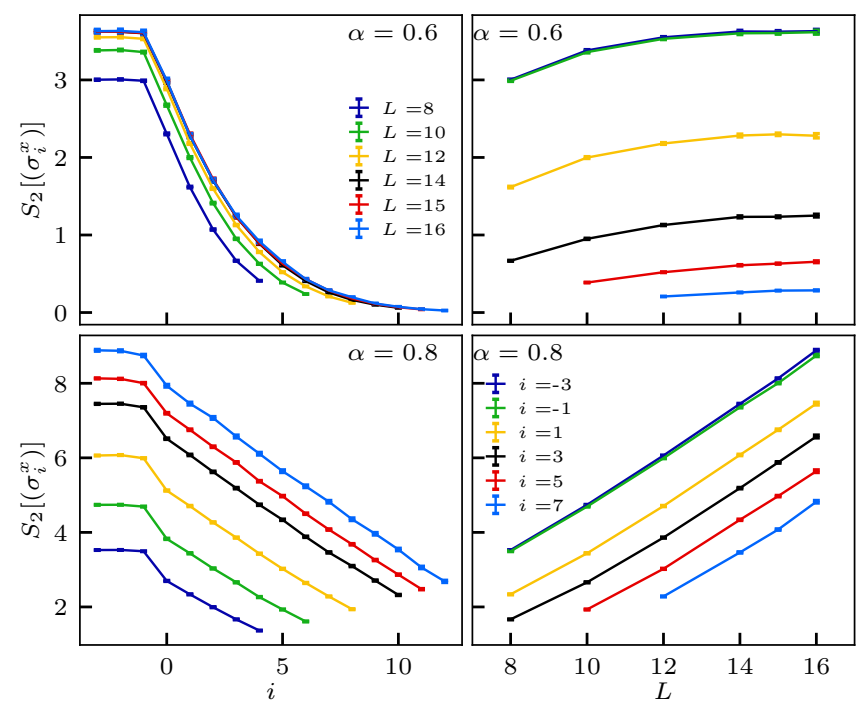

Figure 4. Left panels: Second Rényi entropy $S_{2}$ for excitation created by the operator $\sigma_{i}^{x}$ in an eigenstate. Right panels: evolution of $S_{2}$ at some given sites as a function of the total length $L$.

tribution as $i \geq 0$ increases but no signs of bi-modality, confirming thus the above conclusion. Interestingly, we observe that for all thermalized spins, the distribution departs from a gaussian due to the presence of heavier tails compared to gaussian distributions. This phenomenon has recently been observed in ergodic systems at moderate values of disorder where the dynamics is expected to be sub-diffusive [55, 62].

Participation entropy - Up to here, we have defined the "effective dimension" $d_{i}$ of the Hilbert space only via the spectral function $f(\omega)$. Let us introduce now the participation Rényi entropies $S_{q}$ to obtain a more direct definition:

$$
S_{q}=\frac{1}{1-q} \log \sum_{E}\left|\left\langle E_{0}\left|\sigma_{i}^{x}\right| E\right\rangle\right|^{2 q} .
$$

with $E_{0}$ a state of energy density $\epsilon_{0}$ (at maximal entropy). Here, we will focus on the second Rényi entropy $S_{2}$. Before taking the logarithm, this quantity is analogous to the inverse participation ratio in oneparticle localization[63]. For thermal systems at maximal entropy, $S_{2} \sim L \log 2$ while for localized systems $S_{2} \sim$ const. as a function of the total size $L$. In general, using the ETH (1), we find $S_{2}=-\log \left(\delta \int \mathrm{d} \omega|f(\omega)|^{4}\right)$. If now $f$ is mainly supported on a set of size $\Delta_{i}$ and we use the sum-rule $\int \mathrm{d} \omega|f(\omega)|^{2} \sim 1$ (independent of $L$ ), then we find $S_{2} \approx \log \left(\Delta_{i} / \delta\right)$, which indeed equals $\log d_{i}$ by our definition of $d_{i}$. By comparison with (4) the theory predicts $S_{2} \sim \log (2) L_{\text {therm }}-2|\log (\alpha)| i$, (as long as $\left.S_{2} \geq 0\right)$

We test these predictions for $\alpha=0.6<\alpha_{c}$ and $\alpha=0.8>\alpha_{c}$ in Fig. 4. Our results confirm the behavior
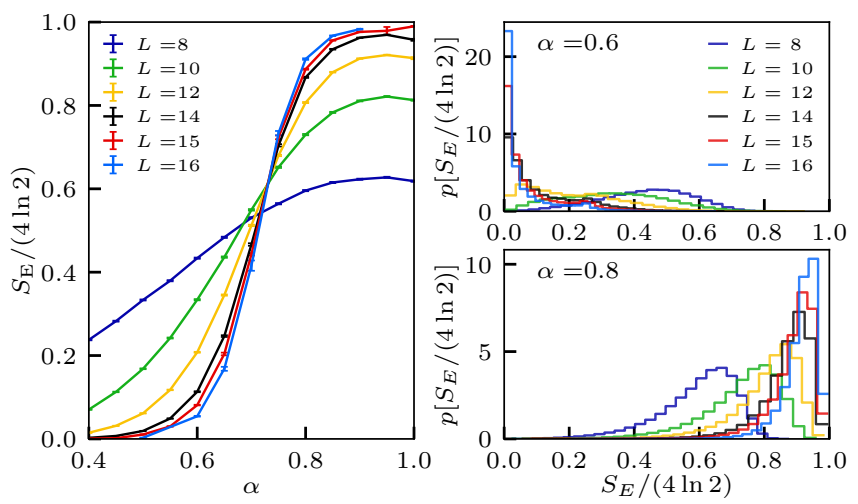

Figure 5. Entanglement entropy of the last 4 l-bits in the chain for different system sizes

that was already commented in the section on local magnetizations. In particular, at $\alpha=0.6$, see Fig. 4 (top), we observe that $S_{2}$ first decreases linearly for small $i \geq 0$ then saturates to a constant value, because $L_{\text {therm }}$ saturates to $L_{c}$. The behavior of $S_{2}$ for a given site is plotted as a function of the total length in the right panels of Fig. 4: $S_{2}$ saturates to a constant value at all sites. For $\alpha=0.8$ instead, $S_{2}$ increases linearly with system size at all sites $i$, and decreases linearly with $i$ for a given size $L$; since the decrease with $i$ is slower than the increase with system size, all spins can be thermalized for arbitrary large system sizes. The evolution of $S_{2}$ at a given site as a function of $L$ shows a linear increase with a slope close to $\log (2)$ as predicted theoretically.

Entanglement entropy - Finally, to have a more direct measurement of the thermalization of the last spins, we compute the entanglement entropy (EE) of the last four spins together for an eigenstate in the middle of the spectrum. If the last four LIOMs remain localized the EE should remain close to 0 , and should converge to 0 as $L$ is increased. Instead, if they are thermalized, the EE should approaches its maximal value $4 \log (2)$. The results are depicted on Fig. 5. In the left panel, we clearly see the trend that, for $\alpha \lesssim 0.7$, the the value of the EE decreases to 0 , while it increases to $4 \log (2)$ for $\alpha \gtrsim 0.7$. This is all the more remarkable since the 4 last l-bits are increasingly far away from the bath with increasing system size. A full distribution is plotted on the right panel for $\alpha=0.6,0.8$ at various lengths, confirming this trend.

Conclusion - We have demonstrated that a small ergodic grain can thermalize an arbitrary number $L_{\text {loc }}$ of localized spins, provided the localization length of the localized spins exceeds the critical value $(2 / \log 2)$. This was achieved numerically by coupling spins with on-site disorder to a GOE system of dimension $8=2^{3}$ with exponentially decreasing couplings. When the localization length is smaller than the critical value, the system drifts towards localization as more and more spins are added. When the localization is above the critical value, the sys- 
tem drifts towards ever cleaner ergodicity as more and more spins are added, even though those spins are coupled very weakly and can not be trivially thermalized.

We would like to thank E. Altman, A. Chandran and J. Imbrie for useful discussions. This project has received funding from the European Union's Horizon 2020 research and innovation programme under the Marie Skłodowska-Curie grant agreement No 747914 (QMBDyn). DJL acknowledges PRACE for awarding access to HLRS's Hazel Hen computer based in Stuttgart, Germany under grant number 2016153659. FH benefited from the support of the projects EDNHS ANR-14-CE250011 and LSD ANR-15-CE40-0020-01 of the French National Research Agency (ANR). WDR acknowledges the support of the Flemish Research Fund FWO and

\section{SUPPLEMENTARY MATERIAL}

Single LIOM - Here, we present additional numerical results for a single LIOM coupled to an ergodic grain of size $L_{b}=3$. Our main focus is to understand how large the direct coupling $g$ has to be in order for the full system to be ergodic. In Fig. 6, we demonstrate that two limiting cases are reached: For large enough coupling $g \gtrsim 0.5$, the distribution of the adjacent gap ratio parameter $r$ follows closely the GOE expectation, which means that the LIOM is well thermalized by the ergodic grain. In the case of completely decoupled LIOM and ergodic grain, the distribution is determined by a "folding" of two independent GOE spectra, denoted as $\mathrm{GOE}^{2}$ in Fig. 6 . Clearly for small coupling strengths, $g \lesssim 0.5$, the distributions are quite close to this case and we conclude that if $g=0.1$, the LIOM is not thermalized by the ergodic region. This strenghtens our argument in the main text that for our longest chains and $\alpha=0.75,0.8,0.85$, the last few spins are coupled so weakly to the ergodic region that they can not be trivially thermalized.

*david.luitz@tum.de

† fhuvenee@ceremade.dauphine.fr

¥ wojciech.deroeck@kuleuven.be

[1] P. W. Anderson, "Absence of Diffusion in Certain Random Lattices," Phys. Rev. 109, 1492-1505 (1958).

[2] L. Fleishman and P. W. Anderson, "Interactions and the Anderson transition," Phys. Rev. B 21, 2366-2377 (1980).

[3] D. M. Basko, I. L. Aleiner, and B. L. Altshuler, "Metalinsulator transition in a weakly interacting many-electron system with localized single-particle states," Annals of physics 321, 1126-1205 (2006).

[4] I. V. Gornyi, A. D. Mirlin, and D. G. Polyakov, "Interacting electrons in disordered wires: Anderson localization and low-t transport," Phys. Rev. Lett. 95, 206603 (2005).

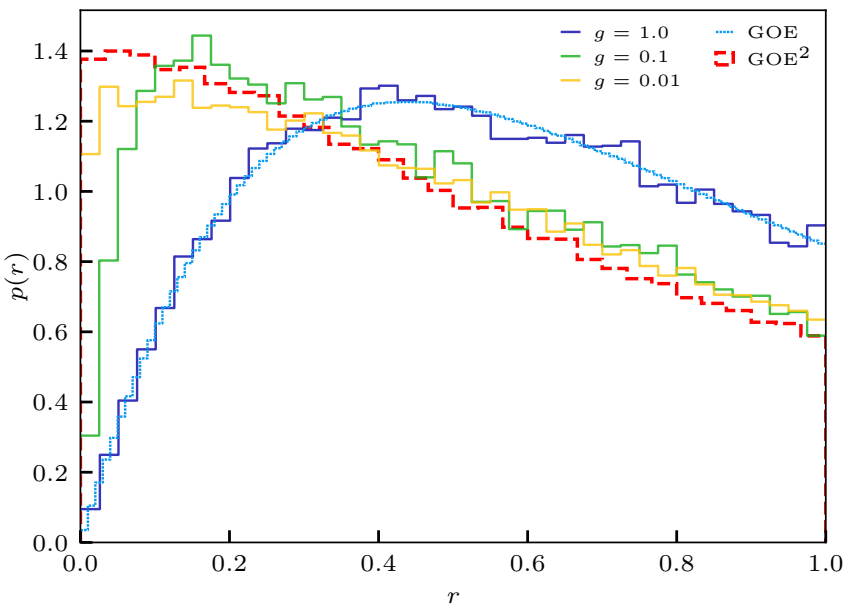

Figure 6. Spectral statistics of a single LIOM coupled to an $L_{b}=3$ ergodic region described by a GOE matrix. For a large enough coupling strength $g \gtrsim 0.5$, the whole system follows the GOE statistics, while for small coupling it reproduces the result expected in the completely uncoupled case $g=0$, where the statistics corresponds to two "folded" GOE spectra.

[5] Marko Žnidarič, Tomaž Prosen, and Peter Prelovšek, "Many-body localization in the Heisenberg $X X Z$ magnet in a random field," Phys. Rev. B 77, 064426 (2008).

[6] Vadim Oganesyan and David A. Huse, "Localization of interacting fermions at high temperature," Phys. Rev. B 75, 155111 (2007).

[7] Arijeet Pal and David A. Huse, "Many-body localization phase transition," Phys. Rev. B 82, 174411 (2010).

[8] David J. Luitz, Nicolas Laflorencie, and Fabien Alet, "Many-body localization edge in the random-field Heisenberg chain," Phys. Rev. B 91, 081103 (2015).

[9] Rahul Nandkishore and David A. Huse, "Many-Body Localization and Thermalization in Quantum Statistical Mechanics," Annual Review of Condensed Matter Physics 6, 15-38 (2015).

[10] Ehud Altman and Ronen Vosk, "Universal Dynamics and Renormalization in Many-Body-Localized Systems," Annu. Rev. Condens. Matter Phys. 6, 383-409 (2015).

[11] Dmitry A. Abanin and Zlatko Papić, "Recent progress in many-body localization," arXiv:1705.09103 (2017), arXiv: 1705.09103.

[12] David J. Luitz and Yevgeny Bar Lev, "The ergodic side of the many-body localization transition," Ann. Phys. (Berlin) (2017), 10.1002/andp.201600350.

[13] Kartiek Agarwal, Ehud Altman, Eugene Demler, Sarang Gopalakrishnan, David A. Huse, and Michael Knap, "Rare-region effects and dynamics near the many-body localization transition," Ann. Phys. (Berlin) (2017), 10.1002/andp.201600326.

[14] S. A. Parameswaran, Andrew C. Potter, and Romain Vasseur, "Eigenstate phase transitions and the emergence of universal dynamics in highly excited states," Ann. Phys. (Berlin) (2017), 10.1002/andp.201600302.

[15] J. Z. Imbrie, V. Ros, and A. Scardicchio, "Review: Local Integrals of Motion in Many-Body Localized systems," arXiv:1609.08076 (2016), arXiv: 1609.08076.

[16] In contrast, Griffiths regions of high disorder are also believed to be responsible for anomalous transport prior 
to the MBL transition [12, 13, 64-69].

[17] John Z Imbrie, "On many-body localization for quantum spin chains," Journal of Statistical Physics 163, 998-1048 (2016).

[18] François Huveneers, "Classical and quantum systems: transport due to rare events," Annalen der Physik, 1600384 (2017).

[19] Wojciech De Roeck and François Huveneers, "Scenario for delocalization in translation-invariant systems," Phys. Rev. B 90, 165137 (2014).

[20] Wojciech De Roeck and François Huveneers, "Asymptotic quantum many-body localization from thermal disorder," Communications in Mathematical Physics 332, 1017-1082 (2014).

[21] Manuel Pino, Lev B. Ioffe, and Boris L. Altshuler, "Nonergodic metallic and insulating phases of josephson junction chains," Proceedings of the National Academy of Sciences 113, 536-541 (2016).

[22] Tarun Grover and Matthew PA Fisher, "Quantum disentangled liquids," Journal of Statistical Mechanics: Theory and Experiment 2014, P10010 (2014).

[23] N. Y. Yao, C. R. Laumann, J. I. Cirac, M. D. Lukin, and J. E. Moore, "Quasi-many-body localization in translation-invariant systems," Phys. Rev. Lett. 117, 240601 (2016).

[24] Mauro Schiulaz and M Müller, "Ideal quantum glass transitions: many-body localization without quenched disorder," AIP Conf. Proc. 1610, 11 (2013).

[25] Mauro Schiulaz, Alessandro Silva, and Markus Müller, "Dynamics in many-body localized quantum systems without disorder," Phys. Rev. B 91, 184202 (2015).

[26] R. M. Nandkishore and S. L. Sondhi, "Many body localization with long range interactions," ArXiv e-prints (2017), arXiv:1705.06290.

[27] François Huveneers, "Drastic fall-off of the thermal conductivity for disordered lattices in the limit of weak anharmonic interactions," Nonlinearity 26, 837 (2013).

[28] D. M. Basko, "Weak chaos in the disordered nonlinear schrödinger chain: destruction of anderson localization by arnold diffusion," Annals of Physics 326, 1577-1655 (2011).

[29] James M. Hickey, Sam Genway, and Juan P. Garrahan, "Signatures of many-body localisation in a system without disorder and the relation to a glass transition," Journal of Statistical Mechanics: Theory and Experiment 2016, 054047 (2016).

[30] Ludovic Berthier and Giulio Biroli, "Theoretical perspective on the glass transition and amorphous materials," Rev. Mod. Phys. 83, 587 (2011).

[31] Ronen Vosk, David A Huse, and Ehud Altman, "Theory of the many-body localization transition in onedimensional systems," Phys. Rev. X 5, 031032 (2015).

[32] Andrew C. Potter, Romain Vasseur, and S. A. Parameswaran, "Universal Properties of Many-Body Delocalization Transitions," Phys. Rev. X 5, 031033 (2015).

[33] David Pekker, Gil Refael, Ehud Altman, Eugene Demler, and Vadim Oganesyan, "Hilbert-Glass Transition: New Universality of Temperature-Tuned Many-Body Dynamical Quantum Criticality," Phys. Rev. X 4, 011052 (2014).

[34] Philipp T. Dumitrescu, Romain Vasseur, and Andrew C. Potter, "Scaling theory of entanglement at the many-body localization transition," arXiv preprint arXiv:1701.04827 (2017).
[35] Thimothee Thiery, Francois Huveneers, Wojciech De Roeck, and Markus Müller, "The MBL/ETH transition: finite and divergent length scales," in preparation.

[36] Vedika Khemani, Say-Peng Lim, D. N. Sheng, and David A. Huse, "Critical properties of the many-body localization transition," Phys. Rev. X 7, 021013 (2017).

[37] Mario Feingold, Nimrod Moiseyev, and Asher Peres, "Ergodicity and mixing in quantum theory. II," Phys. Rev. A 30, 509 (1984).

[38] J. M. Deutsch, "Quantum statistical mechanics in a closed system," Phys. Rev. A 43, 2046 (1991).

[39] Mark Srednicki, "Chaos and quantum thermalization," Phys. Rev. E 50, 888 (1994).

[40] Marcos Rigol, Vanja Dunjko, and Maxim Olshanii, "Thermalization and its mechanism for generic isolated quantum systems," Nature 452, 854-858 (2008).

[41] Ehsan Khatami, Marcos Rigol, Armando Relaño, and Antonio M. García-García, "Quantum quenches in disordered systems: Approach to thermal equilibrium without a typical relaxation time," Phys. Rev. E 85, 050102 (2012).

[42] Luca D'Alessio, Yariv Kafri, Anatoli Polkovnikov, and Marcos Rigol, "From quantum chaos and eigenstate thermalization to statistical mechanics and thermodynamics," Advances in Physics 65, 239-362 (2016).

[43] F. Borgonovi, F. M. Izrailev, L. F. Santos, and V. G. Zelevinsky, "Quantum chaos and thermalization in isolated systems of interacting particles," Physics Reports Quantum chaos and thermalization in isolated systems of interacting particles, 626, 1-58 (2016).

[44] Wojciech De Roeck and François Huveneers, "Stability and instability towards delocalization in many-body localization systems," Phys. Rev. B 95, 155129 (2017).

[45] A bound on the localization length has also been derived in [31], which is entirely different (it holds even without ergodic regions) and for a different quantity (the typical matrix element of the coupling whereas the bound in [44] is for the norm of the coupling). The crucial difference between these bounds is discussed in [35].

[46] Ehud Altman and Dmitry Abanin, "Private communication," (2016).

[47] Gabriele De Chiara, Simone Montangero, Pasquale Calabrese, and Rosario Fazio, "Entanglement entropy dynamics of Heisenberg chains," J. Stat. Mech. 2006, P03001 (2006).

[48] Jens H. Bardarson, Frank Pollmann, and Joel E. Moore, "Unbounded Growth of Entanglement in Models of Many-Body Localization," Phys. Rev. Lett. 109, 017202 (2012).

[49] Maksym Serbyn, Z. Papić, and Dmitry A. Abanin, "Universal Slow Growth of Entanglement in Interacting Strongly Disordered Systems," Phys. Rev. Lett. 110, 260601 (2013).

[50] Maksym Serbyn, Z. Papić, and Dmitry A. Abanin, "Local Conservation Laws and the Structure of the ManyBody Localized States," Phys. Rev. Lett. 111, 127201 (2013).

[51] David A. Huse, Rahul Nandkishore, and Vadim Oganesyan, "Phenomenology of fully many-bodylocalized systems," Phys. Rev. B 90, 174202 (2014).

[52] V. Ros, M. Müller, and A. Scardicchio, "Integrals of motion in the many-body localized phase," Nuclear Physics B 891, 420-465 (2015). 
[53] Louk Rademaker, Miguel Ortuño, and Andres M Somoza, "Many-body localization from the perspective of integrals of motion," Annalen der Physik , 1600322 (2017).

[54] Cecile Monthus, "Many-Body Localization : construction of the emergent local conserved operators via block realspace renormalization," Journal of Statistical Mechanics: Theory and Experiment 2016, 033101 (2016), arXiv: 1509.06258.

[55] David J. Luitz and Yevgeny Bar Lev, "Anomalous Thermalization in Ergodic Systems," Phys. Rev. Lett. 117, 170404 (2016).

[56] Maksym Serbyn, Z. Papić, and Dmitry A. Abanin, "Thouless energy and multifractality across the manybody localization transition," arXiv:1610.02389 (2016).

[57] In well coupled ergodic systems where all local terms are more or less of equal strength, one can think of $\Delta$ as roughly equal to $\epsilon_{0}$, i.e. the energy per site. There is however a glitch in calling this the 'local rate': In ergodic systems with conserved quantities and normal (diffusive) transport, the relaxation time of conserved quantities scales as $L^{2}$ and the associated rate is the Thouless energy. A generic local observable will inherit this slow decay and so its relaxation rate, strictly speaking, is also given by the Thouless energy. Yet, there is a seperation of time scales and for short times, the rate appears to be $\Delta$. In any case, these distinctions do not matter in our work since we focus on the exponential dependence on $L$.

[58] Vadim Oganesyan and David A. Huse, "Localization of interacting fermions at high temperature," Phys. Rev. B 75, 155111 (2007).

[59] The case $\alpha=1$ is particular because the energy interaction between bath and LIOMs becomes much bigger than the bath energy as $L_{l o c}$ grows large. This induces effects $[70,71]$ that are not covered by the theory developed here.

[60] Wouter Beugeling, Roderich Moessner, and Masudul Haque, "Finite-size scaling of eigenstate thermalization," Phys. Rev. E 89, 042112 (2014).
[61] Tatsuhiko N. Ikeda, Yu Watanabe, and Masahito Ueda, "Finite-size scaling analysis of the eigenstate thermalization hypothesis in a one-dimensional interacting bose gas," Phys. Rev. E 87, 012125 (2013).

[62] David J. Luitz, "Long tail distributions near the manybody localization transition," Phys. Rev. B 93 (2016), 10.1103/PhysRevB.93.134201.

[63] Therefore it was called IPR in $[44,56]$.

[64] Yevgeny Bar Lev and David R. Reichman, "Dynamics of many-body localization," Phys. Rev. B 89, 220201 (2014).

[65] Yevgeny Bar Lev, Guy Cohen, and David R. Reichman, "Absence of Diffusion in an Interacting System of Spinless Fermions on a One-Dimensional Disordered Lattice," Phys. Rev. Lett. 114, 100601 (2015).

[66] Kartiek Agarwal, Sarang Gopalakrishnan, Michael Knap, Markus Müller, and Eugene Demler, "Anomalous Diffusion and Griffiths Effects Near the Many-Body Localization Transition," Phys. Rev. Lett. 114, 160401 (2015).

[67] David J. Luitz, Nicolas Laflorencie, and Fabien Alet, "Extended slow dynamical regime close to the manybody localization transition," Phys. Rev. B 93, 060201 (2016).

[68] Marko Žnidarič, Antonello Scardicchio, and Vipin Kerala Varma, "Diffusive and Subdiffusive Spin Transport in the Ergodic Phase of a Many-Body Localizable System," Phys. Rev. Lett. 117, 040601 (2016).

[69] V. Kerala Varma, Alessio Lerose, Francesca Pietracaprina, John Goold, and Antonello Scardicchio, "Energy diffusion in the ergodic phase of a many body localizable spin chain," Journal of Statistical Mechanics: Theory and Experiment 2017, 053101 (2017).

[70] David A. Huse, Rahul Nandkishore, Francesca Pietracaprina, Valentina Ros, and Antonello Scardicchio, "Localized systems coupled to small baths: From anderson to zeno," Phys. Rev. B 92, 014203 (2015).

[71] Rahul Nandkishore and Sarang Gopalakrishnan, "Many body localized systems weakly coupled to baths," Annalen der Physik (2016), 10.1002/andp.201600181. 